\title{
Realisation of analogue divider using current feedback amplifiers
}

\author{
S.-I. Liu
}

J.-J. Chen

Indexing terms: Analogue circuits, Divider circuits

Abstract: New analogue divider circuits using current feedback amplifiers (CFAs) are presented. Basically, they consist of two CFAs, two MOS transistors biased in the triode region, and a resistor. Furthermore, an integrable divider circuit is also presented. Experimental results are given to demonstrate the feasibility of the proposed circuits. The proposed divider will be useful in high-frequency analogue signal processing.

\section{Introduction}

An analogue divider is an important building block in analogue computation, fuzzy control and instrumentation, etc. [1-3]. Many analogue continuous-time and sampled-data divider circuits have been presented in the literature [4-8]. Most of them have used conventional operational amplifiers as building blocks to synthesise the division function. However, the finite-gain-bandwidth product of the operational amplifiers will limit the highfrequency operation and accuracy of the divider.

Current-mode circuits have been receiving significant attention in analogue signal processing circuits [9]. A new amplifier called the current feedback amplifier (CFA) $[10,11]$ has been realised to circumvent the finite-gainbandwidth limitation of the conventional operational amplifier. It can provide not only constant bandwidth independent of the closed-loop gain but also high slew rate (i.e. $2000 \mathrm{~V} / \mu \mathrm{s}$ ) [11]. It can be also used as a secondgeneration current conveyor (CCII) [12] by using its compensation node [13]. Moreover, since a CFA was built with a voltage buffer, it can drive external loads easily. In this paper we propose new analogue division circuits using CFAs. Experimental results are also given to verify the theoretical analysis.

\section{Circuit description}

The simplified equivalent circuit and symbol for a current feedback amplifier (CFA) are shown in Fig. $1 a$ and $b$, respectively. This circuit is equivalent to a $\mathrm{CCII}$ with a

(C) IEE, 1995

Paper 1609 G (E10), received 23rd February 1994

S.-I. Liu is with the Department of Electrical Engineering, National Taiwan University, Taipei, Taiwan 10664, Republic of China, on leave from the National Taiwan Institute of Technology

J.-J. Chen is with the Department of Electrical Engineering, National Taiwan University, Taipei, Taiwan 10664, Republic of China

IEE Proc.-Circuits Devices Syst., Vol. 142, No. 1, February 1995

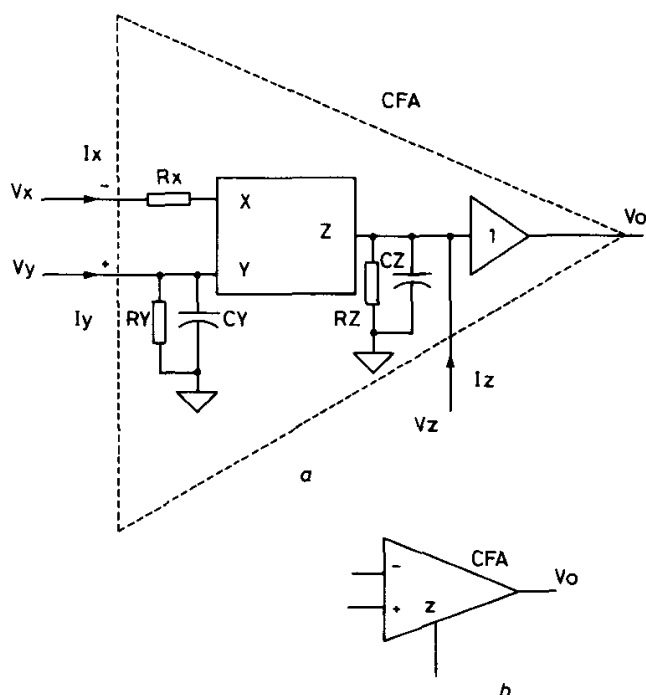

Fig. 1 Equivalent circuit for a current feedback amplifier

voltage buffer [12]. Its characteristics can be modelled as $\left[\begin{array}{l}v_{x} \\ i_{y} \\ i_{z}\end{array}\right]=\left[\begin{array}{lll}1 & 0 & 0 \\ 0 & 0 & 0 \\ 0 & 0 & 1\end{array}\right]\left[\begin{array}{l}v_{y} \\ v_{z} \\ i_{x}\end{array}\right]$ and $v_{0}=v_{z}$

The proposed analogue division circuit with two CFAs is shown in Fig. 2. It consists of two CFAs, two NMOS transistors biased in the triode region and a resistor. The drain current of an NMOS transistor biased in the triode region can be expressed by [14]

$$
I_{D}=F\left(V_{D}, V_{G}\right)-F\left(V_{S}, V_{G}\right)
$$

with

$$
\begin{gathered}
\begin{aligned}
& F\left(V_{X}, V_{G}\right)= 2 K\left(V_{G}-V_{B}-V_{F B}-\phi_{B}\right) V_{X} \\
&-K\left(V_{X}-V_{B}\right)^{2}-\frac{4}{3} K \gamma\left(V_{X}-V_{B}+\phi_{B}\right)^{3 / 2} \\
& K=\frac{W}{2 L} \mu C_{o x} \quad \gamma=\frac{\sqrt{ }\left(2 q N_{A} \varepsilon_{s}\right)}{C_{o x}} \quad V_{X}=V_{D} \text { or } V_{S}
\end{aligned}
\end{gathered}
$$

where $I_{D}$ is the drain current in the triode region, $W$ and $L$ are the channel length and width, respectively, $\mu$ is the effective mobility, $V_{F B}$ is the flatband voltage, $N_{A}$ is the 
substrate doping concentration, $C_{o x}$ is the gate oxide capacitance per unit area, $\phi_{B}$ is the approximate surface potential in strong inversion, and $V_{G}$ and $V_{B}$ are the gate

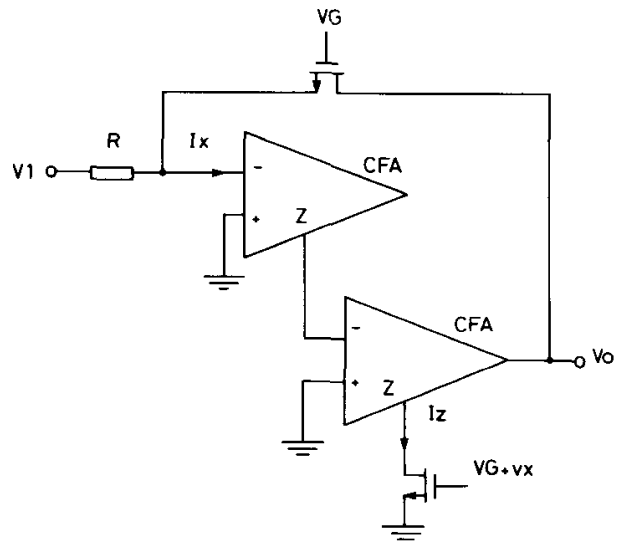

Fig. 2 Proposed divider circuit

and substrate voltages, respectively. Let us examine the currents $i_{x}$ and $i_{z}$ in Fig. 2 . Routine circuit analysis yields

$$
\begin{aligned}
& i_{x}=\frac{v_{1}}{R}+F\left(v_{0}, V_{G}\right)-F\left(0, V_{G}\right) \\
& i_{z}=F\left(v_{0}, V_{G}+v_{x}\right)-F\left(0, V_{G}+v_{x}\right)
\end{aligned}
$$

Thus, the output voltage of this divider can be expressed as

$$
v_{o}=\frac{v_{1}}{2 K R v_{X}}
$$

The even and odd nonlinearities of the MOS transistors have been cancelled. To keep the MOS transistor in the triode region, the following condition should be satisfied:

$$
\min \left(V_{G}, V_{G}+v_{X}\right)>v_{o}+V_{T}
$$

where $V_{T}$ is the threshold voltage of the MOS transistor. To consider the stability of the proposed divider circuit, assume that there is a parasitic capacitor $C_{z}$ at the compensation node $Z$ of the CFA. One can rewrite eqn. 4 as

$$
v_{o}=\frac{v_{1}}{R\left(2 K v_{X}+s C_{z}\right)}
$$

Hence, for the divider circuit to be stable, the pole of eqn. 6 should be in the left half-plane (LHP). That is,

$$
v_{X}>0
$$

Similarly, one can obtain another divider circuit by exchanging the gate voltages $V_{G}$ and $V_{G}+v_{x}$ of Fig. 2 . Furthermore we can use MOS transistors biased in the triode region to replace the passive resistor $[4,14,15]$. The proposed integrable divider was shown in Fig. 3. We can express the currents $i_{x}$ and $i_{z}$ in Fig. 3 as

$$
\begin{aligned}
i_{x}= & F\left(v_{1}, V_{G A}\right)-F\left(0, V_{G A}\right) \\
& +F\left(v_{o}, V_{G A}\right)-F\left(0, V_{G A}\right)
\end{aligned}
$$

$$
\begin{aligned}
i_{z}= & F\left(v_{1}, V_{G B}\right)-F\left(0, V_{G B}\right) \\
& +F\left(v_{o}, V_{G A}+v_{x}\right)-F\left(0, V_{G A}+v_{x}\right)
\end{aligned}
$$

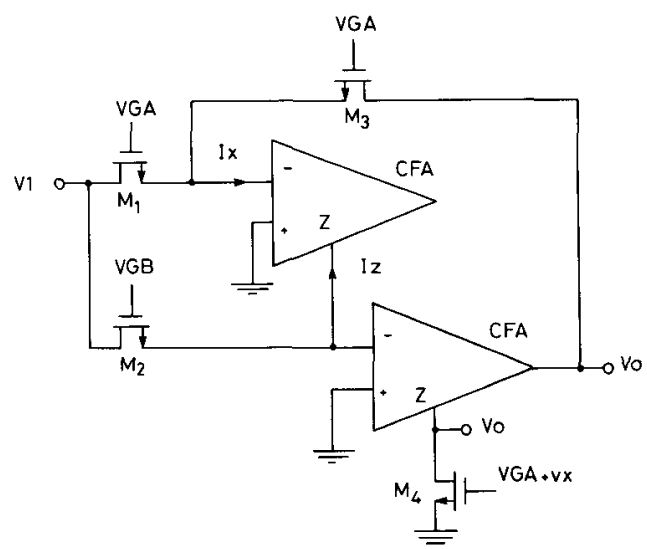

Fig. 3 Integrable analogue divider circuit

Routine circuit analysis yields

$$
v_{o}=\frac{K_{I}\left(V_{G A}-V_{G B}\right)}{K_{o} v_{X}} v_{1}
$$

where $K_{I}$ is the transconductance parameter of $M_{1}$ and $M_{2}$ and $K_{o}$ is that of $M_{3}$ and $M_{4}$. Because all devices have been assumed to be in the triode region, eqn. 10 only holds when

$$
v_{1}, v_{0} \leqslant \min \left[V_{G A}-V_{T}, V_{G B}-V_{T}, V_{G A}+v_{x}-V_{T}\right]
$$

The proposed divider circuit will be suitable for monolithic integration.

\section{Experimental results}

The characteristics of a nonideal CFA with the highimpedance input grounded can be given by

$$
\begin{aligned}
& i_{z}=\alpha i_{x} \\
& v_{0}=\beta v_{z}
\end{aligned}
$$

where $\alpha=1-\varepsilon_{1}$ and $\varepsilon_{1}\left(\varepsilon_{1} \ll 1\right)$ and $\beta=1-\varepsilon_{2}$ and $\varepsilon_{2}$ $\left(\varepsilon_{2} \ll 1\right)$ denote the current tracking error and voltage tracking error, respectively. Assume that the CFAs in Fig. 2 have the same current transfer ratio $\alpha$. A detailed analysis for the transfer function of Fig. 2 yields

$$
v_{o} \cong \frac{\alpha^{2} \beta \frac{v_{1}}{2 K R}}{\left(V_{G}-V_{T}\right)\left(1-\alpha^{2} \beta\right)+v_{X}}
$$

The current and voltage tracking errors will result in the denominator $v_{x}$ with an offset voltage in the interesting frequency range. To demonstrate the proposed circuits, we breadboard the circuits by using commercial CFAs (AD844) and CMOS transistor arrays (CD4007). The power supply is $\pm 12 \mathrm{~V}, V_{G}=7.8 \mathrm{~V}$ and $R=5.1 \mathrm{k} \Omega$. The $D C$ transfer functions were measured and shown in Fig.

IEE Proc.-Circuits Devices Syst., Vol. 142, No. I, February 1995 
$4 a$ and $b$. The horizontal variable is $v_{x}$ and the vertical one is $v_{0}$. Fig. $4 a$ and $b$ show the $v_{o}-v_{x}$ transfer curve with $v_{1}=1.1 \mathrm{~V}$ and $v_{1}=-1.1 \mathrm{~V}$, respectively. A ramp

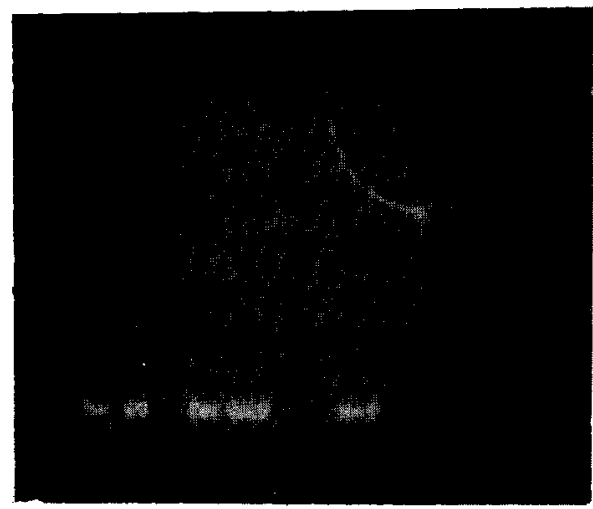

$a$

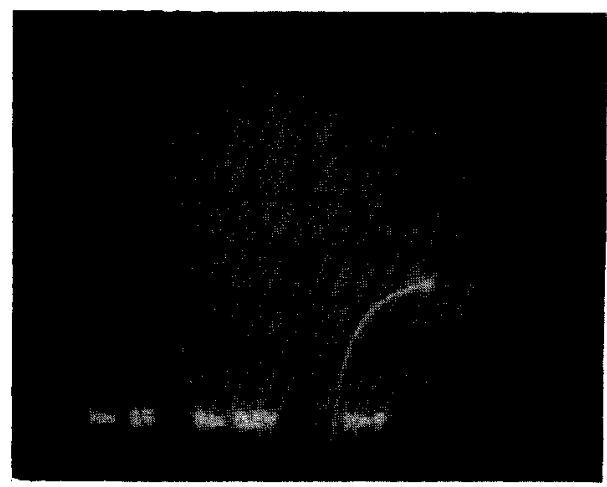

Fig. 4 Transfer functions $a \mathrm{DC}$ transfer function ( $v_{0}$ against $v_{x}$ ) of proposed divider circuit with $v_{1}=1.1 \mathrm{~V}$,
$V_{G}=7.8 \mathrm{~V}$ and $R=5.1 \mathrm{k} \Omega$. Horizontal scale is $1 \mathrm{~V} /$ div and vertical scale is $0.2 \mathrm{~V} /$ div

$b$ DC transfer function ( $v_{o}$ against $v_{x}$ ) of proposed divider circuit with $v_{1}=$ $b$ DC transfer function $\left(v_{o}\right.$ against $\left.v_{x}\right)$ of proposed divider circuit with $v_{1}=$
$-1.1 \mathrm{~V}, V_{G}=7.8 \mathrm{~V}$ and $R=5.1 \mathrm{k} \Omega$. Horizontal scale is $1 \mathrm{~V} / \mathrm{div}$ and vertical scale

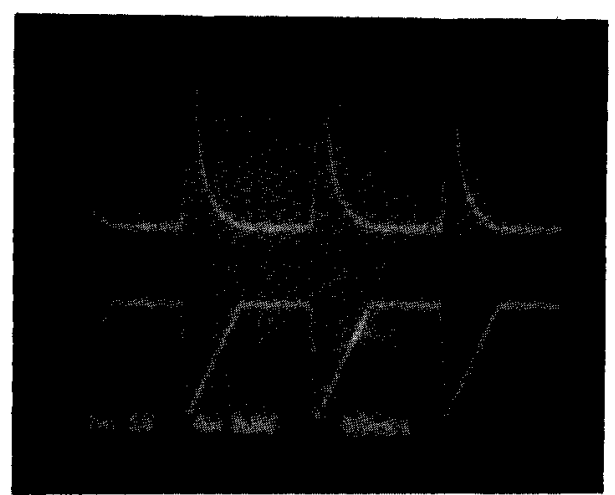

Fig. 5 Ramp signal and output voltage

Lower trace (1 V/div) is ramp signal of $v_{x}$ and upper trace $(0.2 \mathrm{~V} / \mathrm{div})$ is output voltage $\mathrm{V}_{0}$ of divider. Horizontal scale is $0.5 \mathrm{~ms} / \mathrm{div}$

IEE Proc.-Circuits Devices Syst., Vol. 142, No. 1, February 1995 signal with a positive slope was applied to $v_{X}$, and Fig. 5 shows the time domain response of the proposed divider circuit. The frequency response of the proposed divider
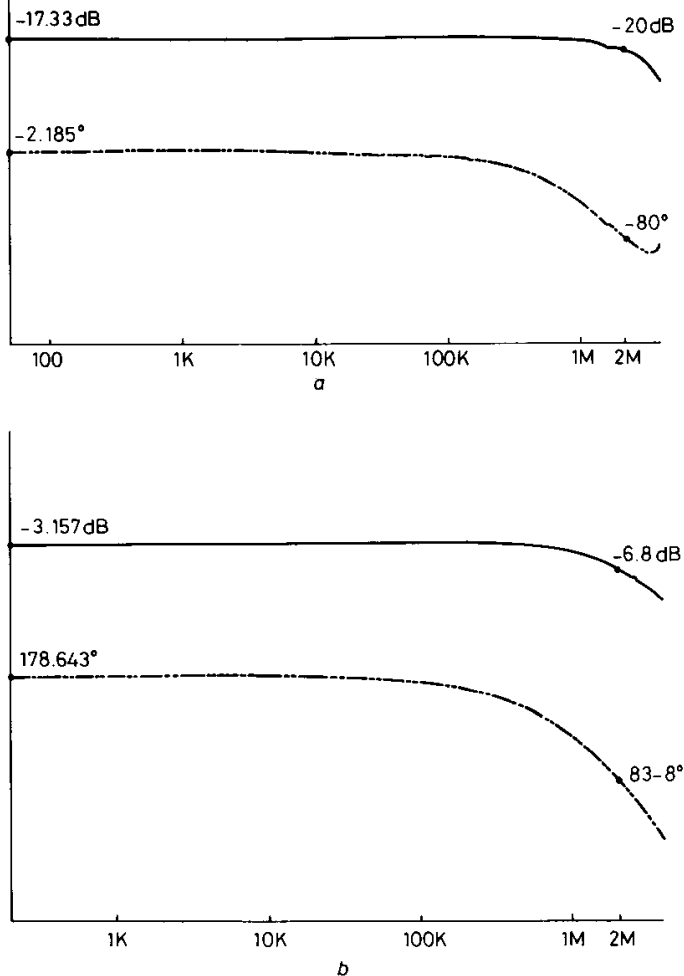

Fig. 6 Gain and phase responses

a Gain $(10 \mathrm{~dB} / \mathrm{div})$ and phase $\left(45^{\circ} / \mathrm{div}\right)$ responses of proposed divider $\left(v_{\sigma} / v_{1}\right)$ with constant $v(=1.1 \mathrm{~V})$. Upper trace is gain response and lower one is phase response $b$ Gain $(10 \mathrm{~dB} / \mathrm{div})$ and phase $\left(45^{\circ} / \mathrm{div}\right)$ responses of proposed divider $\left(v_{0} / v_{x}\right)$ with constant $v_{1}(=1.1 \mathrm{~V})$. Upper trace is gain response and lower one is phase response

circuit was also measured in Fig. $6 a$ and $b$. Fig. $6 a$ shows the frequency response of $v_{1}$ against $v_{0}$ with $v_{x}=1.1 \mathrm{~V}$. Its $-3 \mathrm{~dB}$ bandwidth was about $2 \mathrm{MHz}$. Fig. $6 b$ shows the frequency response of $v_{x}$ against $v_{0}$ with $v_{1}=1.1 \mathrm{~V}$. Its $-3 \mathrm{~dB}$ bandwidth was about $1.8 \mathrm{MHz}$.

\section{Conclusions}

New analogue division circuits using CFAs have been proposed. Experimental results are given to demonstrate the feasibility of the proposed circuits. The divider circuit has a simple structure. Frequency responses show that the proposed divider will be useful in high-frequency analogue signal processing applications.

\section{References}

1 MEAD, C., and ISMAIL, M.: 'Analog VLSI implementation of neural systems' (Kluwer Academic, Boston, 1989) 
2 SHEINGOLD, D.H. (Ed.): 'Nonlinear circuit handbook' (Analog Devices, Norwood, MA, 1974)

3 YAMAKAWA, T.: 'High-speed fuzzy controller hardware system: the Mega-FIPS machine', Inf. Sci., 1988, 45, pp. 113-128

4 KHACHAB, N.I., and ISMAIL, M.: 'A nonlinear CMOS analog cell for VLSI signal and information processing', IEEE $J$. SolidState Circuits, 1991, 26, pp. 1689-1698

5 GHOSH, D., and PATRANABIS, D.: 'A simple analog divider having independent control of sensitivity and design condition', IEEE Trans., 1990, IM-39, pp. 522-526

6 LAOPPOULOS, Th. L., and KARYBAKAS, C.A.: 'A simple analog division scheme', IEEE Trans., 1991, IM-40, pp. 779-782

7 ARONHIME, P., DESAI, M., and STEPHENS, J.: 'Quotient circuits employing VVR', IEEE Trans., 1992, IM-41, pp. 679-684

8 LIU, S.I., WU, D.S., TSAO, H.W., WU, J., and TSAY, J.H.: 'Nonlinear circuit applications with current conveyors', IEE Proc. G, 1993,140 , pp. 1-6
9 WILSON, B.: 'Recent developments in current conveyors and current-mode circuits', IEE Proc. G, 1990, 137, (2), pp. 63-77

10 'Linear products data book' (Analog Devices, Norwood, MA, 1990)

11 'Designer's guide for 200 series op amp'. Application note 200-1 (Comlinear Corporation, 4800 Wheaton Drive, Ft. Collins, CO 80525 , November 1984)

12 SEDRA, A., and SMITH, K.C.: 'A second generation current conveyor and its applications', IEEE Trans., 1970, CT-17, pp. 132-134

13 SVOBODA, J.A., MCGORY, L., and WEBB, S.: 'Applications of a commercially available current conveyor', Int. J. Electron., 1991, pp. 159-164

14 BANU, M., and TSIVIDIS, Y.: 'Detailed analysis of nonlinearities in MOS fully integrated active RC filters based on balanced networks', IEE Proc. G, 1984, 134, pp. 190-196

15 ZARNUL, C.: 'Novel MOS resistive circuit for synthesis of fully integrated continuous-time filters', IEEE Trans., 1986, CAS-33, pp. 718-721 